\title{
A Case Report on Efavirenz Induced Gynaecomastia
}

\author{
Rajalakshmi Rukmangathen ${ }^{*}$, Vasundara Devi Brahmanapalli², Meghana Devulapalli³, Durga Prasad \\ Thammisetty ${ }^{4}$, Avanthi Bondalapati ${ }^{3}$, Shabana Banu Shaik ${ }^{3}$, Pavan Kumar Bathula ${ }^{3}$
}

${ }^{1}$ Patient Safety Pharmacovigilance Associate (Pharmacovigilance Programme of India), ADR Monitoring Centre (AMC), S.V. Medical College, Tirupathi, Andhra Pradesh, INDIA.

${ }^{2}$ Co-ordinator AMC, Professor \& Head of the Department of Pharmacology, S. V. Medical College, Tirupathi, Andhra Pradesh, INDIA. ${ }^{3}$ Student, Doctor of Pharmacy, Department of Pharmacy Practice, Sri Padmavathi School of Pharmacy, Tiruchanoor, Andhra Pradesh, INDIA.

${ }^{4}$ Assistant Professor, Department of Pharmacy Practice, Sri Padmavathi School of Pharmacy, Tiruchanoor, Andhra Pradesh, INDIA.

\begin{abstract}
Gynaecomastia in simple terms means development of breast in the males. This may be associated with various clinical conditions in men depending on the age at the time of diagnosis and may be due to intake of certain drugs that have the potential to cause breast enlargement. In HIV positive individuals Antiretro viral agent's intake was associated with development of breast tissue in males with efavirenz being one of such drugs. We report a case of efavirenz induced gynaecomastia in 37 year old male seropositive patient.
\end{abstract}

Key words: Breast masses, Antiretroviral therapy, Drug induced gynaecomastia, Fine Needle Aspiration Cytology, Seropositive, Breast enlargement, Efavirenz, Gynaecomastia.

\section{INTRODUCTION}

Gynaecomastia is clinical condition associated with the development of the breast tissue in males. ${ }^{1}$ The histological findings of the breast enlargement reveal excess growth of the glandular of the male breast which is not accompanied by malignancy. ${ }^{2}$ The growth of breast tissue can be attributed to an increase in ductal tissue and periductal stroma. ${ }^{1}$ Yet another significantly demonstrable feature of gynaecomastia was the presence of excess amounts of fibrous connective tissue stroma with propagation of ducts. ${ }^{3}$

In gynaecomastia, the tissue is usually soft, elastic or firm. Upon clinical examination, findings reveal a rubbery texture that extends from the nipple concentrically and the size of the enlarged mass is greater than $0.5 \mathrm{~cm}$ in diameter. ${ }^{2}$ In about $50 \%$ of the affected individuals gynaecomastia tends to bilateral., ${ }^{2,3}$ Over a period the fibrotic connective tissue becomes more fibrous, denser and hard. ${ }^{3}$ So, it is essential to differentiate the underlying condition from breast cancer. Generally, breast cancer is not uncommon in males and breast enlargement tends to be hard, firm, and unilateral along with nipple discharge and axillary lymphadenopathy. ${ }^{2}$

Commonly breast enlargement is observed in different age groups such as in neonates, adolescents, and in older men. ${ }^{2}$ The prevalence of gynaecomastia in adults varies from $24-70 \%$ with presentation ranging between the ages of $50-80$ year. ${ }^{2,3}$ The major factor associated with this might be due to the presence of underlying clinical conditions/ comorbid illnesses making an individual more susceptible to histological and clinical breast changes. ${ }^{2,3}$ If Human Immunodeficiency Virus (HIV) and Acquired Immunodeficiency Syndrome (AIDS) were to considered as the comorbidity, for the breast enlargement then the possible mechanisms linked to this might be either immunodeficiency or drug induced alteration leading to a spectrum of varied diagnosis such as gynaecomastia, lipomastia, pseudoangiomatous stromal hyperplasia, and reactive lymphoid tissue hyperplasia causing
DOI: 10.5530/ijopp.10.3.45

Address for correspondence: Rajalakshmi Rukmangathen, Patient safety Pharmacovigilance Associate (Pharmacovigilance programme of India), ADR Monitoring Centre (AMC), S.V. Medical College, Tirupathi-517507, Andhra Pradesh, INDIA. Phone No: 9985795730 E-mail Id: rrajalakshmi2002@ yahoo.in

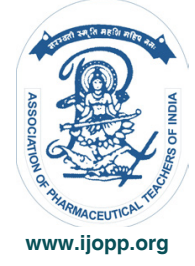


intramammary lymphadenopathy. ${ }^{4}$

The increase in incidence of breast enlargement in seropositive HIV/ AIDS individuals is linked up with antiretroviral therapy (ART). At times, ART may also cause lipomastia, a condition with excess deposition of adipose tissue in the breast. ${ }^{4}$ So, it is necessary to differentiate lipomastia from gynaecomastia by physical examination. $^{2}$

\section{CASE DETAILS}

A 37 year old male seropositive patient on complaints of breast tenderness and pain for 3 months. His medical and medication history is as follows.

The patient was diagnosed to be positive for HIV 10 years back with a CD 4 count of 424 cells $/ \mathrm{mm}^{3}$. A year later due to a fall in the CD4 count to 155 cells $/ \mathrm{mm}^{3}$ the patient was initiated with zidovudine, lamivudine and nevirapine (ZLN) therapy. After 2 months of ZLN therapy the patient experienced a first spell of drop in haemoglobin $(\mathrm{Hb})$ to $4.2 \mathrm{~g} / \mathrm{dL}$. So, in view of this, the patient was changed to stavudine, lamivudine and nevirapine (SLN) based regimen. After 2 months on restoration of $\mathrm{Hb}$ levels to $11.8 \mathrm{~g} / \mathrm{dL}$ the patient was again restarted with ZLN regimen.

The therapy with ZLN was continued for a period of 9 years without any further complaints and then the patient experienced a second spell of anaemia with a reduction in the $\mathrm{Hb}$ levels to $4.4 \mathrm{~g} / \mathrm{dL}$. About this the patient was changed to tenofovir, lamivudine and efavirenz (TLE) based regimen. The patient had good immunologic response to the changed regimen with a CD4 count of 993 cells $/ \mathrm{mm}^{3}$. Despite of improvement in the CD4 counts the patient experienced a discomfortness associated with pain and tenderness in the left breast after 10 months of therapy with TLE.

Upon physical examination patient was found to have a breast mass Figure 1. So, to further evaluate the condition ultrasonography (USG) and fine needle aspiration cytology (FNAC) was performed. The USG depicted a normal right breast and mild relative hypertrophy of the fibroglandular tissue suggesting a mammography corelation where as the FNAC supported the findings of USG revealing branching sheets and clusters of benign looking ductal epithelial cells with haemorrhage, focal fibromyxoid stroma cells and scant blood elements in the background indicating the presence of gynaecomastia. Apart from these, no other laboratory investigations were performed.

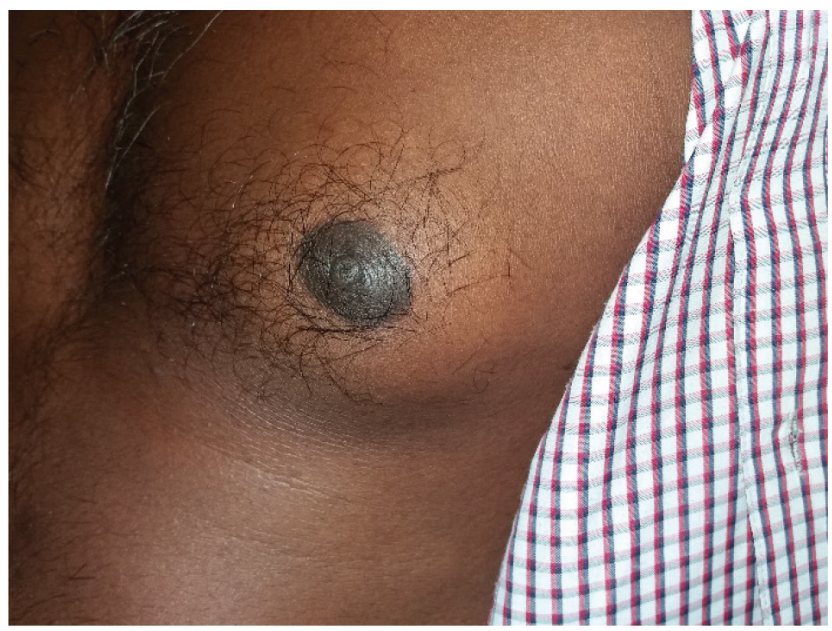

Figure 1: Breast mass on left side.

\section{DISCUSSION}

The incidence of gynaecomastia in men with HIV seropositive status taking highly active antiretroviral therapy (HAART) was found to be $0.8 / 100$ patient years. The prevalence was found to be about $2.8 \%$ in whom the average treatment duration was 3 years. ${ }^{2,5}$ In a study by PAM et al, analysis of fine needle aspiration (FNA) of breast masses in HIV infected patients revealed that $83 \%$ were diagnosed to have gynaecomastia, among them $40 \%$ were using HAART. ${ }^{4}$

Gynaecomastia in a HIV positive individual can be attributed to direct factors involving pathophysiological alterations such as hypogonadism, increased production of prolactin and presence of HIV associated cirrhosis. Apart from the direct factors, indirect factors such as ART consisting of protease inhibitors such as Saquinavir, nucleoside reverse transcriptase inhibitors such as Stavudine, Zidovudine, and Lamivudine and antifungal such as Ketoconazole were also contributing to the development of breast masses in HIV patients. ${ }^{4,5}$ So, it is understandable that an interplay of both direct and indirect factors can predispose to the development of gynaecomastia.

In the present case, it was evident from the past medical and medication history that the patient had no complaints of breast tenderness and breast enlargement with the previously used regimens such as ZLN and SLN. The patient experienced symptoms and associated discomfort after being treated with TLE regimen for a period of 13 months. Of the other two existing drugs in the current regimen, Efavirenz is likely to cause gynaecomastia which was like that reported by Oche et.al., and Ishwar et al. ${ }^{6,7}$

The exact mechanism of EFV induced gynaecomastia was not well known but various hypothesis was

Indian Journal of Pharmacy Practice, Vol 10, Issue 3, Jul-Sep, 2017 
formulated such as a direct oestrogenic effect, stimulation of an immune response and cytochrome P450 induced changes in the steroid metabolism. ${ }^{8}$ The underlying mechanisms were a decrease in the oestrogen metabolism, displacement from oestrogen binding globulin and diminished testosterone biosynthesis. ${ }^{7}$

Firstly, the direct oestrogenic effect of EFV was explained through a series of experimental models by MJ Shikora et al, In their experimental study it was proved that EFV can induce the growth of breast cancer cell lines MCF-7 and ZR-75-1 which have oestrogen receptor (ER) positivity. This growth effect was also reversed by ICI 182,780 cell line that has anti-oestrogenic effect. It was also proved that EFV can bind to ER-alpha (ER-a). These effects were observed in the cell model at the dose of $600 \mathrm{mg}$ of daily administration of EFV with a mean steady state minimum of $5.6 \mathrm{~mm}$ and a maximum concentration of $12.9 \mathrm{~mm}$ which was within the therapeutic range. ${ }^{8}$

Secondly, regaining the suppressed immune responses on taking HAART was proposed to be the underlying hypothesis. In this condition, there will be an enhancement in the T-helper cell mediated cytokine responses with an elevation in the interleukin-2, 6 (IL-2, IL-6) levels. Of them, IL-2 was associated with the proliferation of human carcinoma cells of the breast in vitro and IL-6 was involved in elevating the aromatase activity resulting in an increase in the oestrogen levels in the breast tissue leading to development of breast masses. ${ }^{2,5,6}$

Lastly, the antiretroviral agents cause the inhibition of the cytochrome P-450 enzymes causing a drastic change in the oestrogen-androgen ratio. It was also found that EFV a potent inhibitor of cytochrome P-450, when given along with ethynil estradiol resulted in a $37 \%$ increase in the area under curve (AUC) of the drug, thus causing a prominent elevation in the oestrogen levels. ${ }^{1}$ Thus these mechanisms influence the occurrence of gynaecomastia in a HIV positive individual taking HAART.

In this case, after confirming the diagnosis of gynaecomastia the patient was kept under observation to monitor an increase in the size of the breast mass as certain breast masses tend to resolve spontaneously. The therapy with TLE was being continued in the patient and no specific therapeutic agents were given to treat the condition. This was because the breast masses reverted over a period and were not painful as reported by Kenji et al, as therapy with TLE was very effective first line management keeping in view of the patient's exposure previously to the other HAART regimens. ${ }^{1}$

But, a few studies conducted demonstrated that EFV induced gynaecomastia can be treated with $20 \mathrm{mg}$ of tamoxifen which was widely used for the prophylaxis and management of anti-androgen-induced gynaecomastia. ${ }^{8}$ Apart from tamoxifen another oestrogen receptor inhibitor raloxifene, and anastrazole an aromatase enzyme inhibitor has been used in the used in the management of gynaecomastia. Tamoxifen at the dose of $10-20 \mathrm{mg} /$ day and raloxifene at the dose of $60 \mathrm{mg} /$ day for 3-9 months were used. Anastrazole was indicated in the individuals with aromatase excess. ${ }^{3}$

However, if gynaecomastia continues to pursue for a period of more than 1-2 years then surgical management seems to be most effective compared to therapeutic management. The approaches for surgeries include surgical excision through Webster's or endoscopic methods. Removal of glandular tissue using an endoscopic cartilage shaver has good results without any scars. A newly available method such as radiofrequencyassisted liposuction removes both the fat and the glandular tissue. The other technique that is associated with slight invasion includes an ultrasound guided using an 8-guage mammotomy biopsy system. ${ }^{9}$

Although various approaches exist for the management of gynaecomastia, it is advisable to seek patient's opinion prior to initiating any management strategy. The patient should be given a choice to select a suitable and effective means of treatment by making him aware of the possible outcomes of the therapy. To improve the accuracy of our assessment, causality assessment was undertaken using the WHO casuality Assessment scale and the likelihood of the reaction was possible.

\section{CONCLUSION}

Gynaecomastia due to drugs is relatively rare when compared to the underlying alterations in the pathophysiologic conditions. The incidence of gynaecomastia was increasing in the men with HAART therapy. So, it is necessary to identify the patients with increased risk and educate them regarding the clinical condition. It is of utmost importance to identify the exact mechanisms underlying the disease so that an appropriate therapeutic intervention can be made without exposing the patient to unnecessary medications and without heading towards a surgical approach.

\section{ACKNOWLEDGEMENT}

We thank Principal, Sri Venkateswara Medical College and ART centre, SVRRGG hospital, Tirupati. We also express our sincere thanks for technical \& logistic support 
of NCC-PvPI, Indian Pharmacopoeia Commission, Ghaziabad.

\section{CONFLICT OF INTEREST}

The authors declare no conflict of interest.

\section{ABBREVIATION USED}

HIV: Human Immunodeficiency Virus; AIDS: Acquired Immunodeficiency Syndrome; ART: Antiretroviral Therapy; ZLN: Zidovudine, lamivudine and nevirapine; TLE: Tenofovir, lamivudine and efavirenz; Hb: Hemoglobin; SLN: stavudine, lamivudine and nevirapine; USG: ultrasonography; FNAC: Fine needle aspiration cytology; HAART: Highly active antiretroviral therapy; ER: oestrogen receptor; AUC: Area under curve

\section{REFERENCES}

1. Kenji U, Mitsuru K, Eilchiro Y, Kei K, Kei M, Koichi M, Keiichi M. A case of gynaecomastia associated with efavirenz. Journal of Nara Medical Association. 2007:141-5.

2. Ari E, Adrian D. Drug-induced gynaecomastia. Drug Saf. 2008;7(6):691-702.

3. Harold EC. Approach to the patient with Gynaecomastia. J Clin Endocrinol Metab. 2011;96(1): 15-21.

4. Pam M, Bruce JD, Liron P. Fine Needle Aspiration of Breast Masses in HIVInfected Patients. Cancer Cytopathology. 2010; DOI: 10.1002/cncy.20083:218-24.

5. Fnu D, Glenn DB. Drug-induced gynecomastia: an evidence-based review. Drug Saf. 2012;11(5):779-95

6. Oche OA, Patricia AA, Peter NE, Halima MS, Raphael OO, Eunice A, et al. Efavirenz-induced gynaecomastia in HIV-infected Nigerian men: A report of six cases. Journal of Medicine and Medical Sciences. Nov 2011;2:1221-4.

7. Ishwar SH, Mahabaleshwar SM, Basith LK, Vinaykumar BH. Efavirenz-Induced Gynaecomastia in HIV Infected Males: A Report of 2 Cases. J. Evid. Based Med. Healthc. 2016;3:3729-32.

8. Sikora MJ, Rae JM, Johnson MD, Desta Z. Efavirenz directly modulates the oestrogen receptor and induces breast cancer cell growth. HIV Medicine. 2010;11(9):603-7.

9. Anders F, Richard L, Guglielmo R, Anna E, Fabio S, Gennaro S. Gynecomastia: A systematic review. J Plast Surg Hand Surg. 2015;49(6):311-8. DOI: 10.3109/2000656X.2015.1053398:1-8 